\title{
Reservoir characterization of Habiganj gas field
}

\author{
Islam Md. Shofiqul*, Jahan Labiba Nusrat \\ Department of Petroleum and Mining Engineering, Shahjalal University of Science and Technology, Sylhet 3114, Bangladesh
}

Email address:

sho_fiq@yahoo.com (Islam M. S.)

\section{To cite this article:}

Islam Md. Shofiqul, Jahan Labiba Nusrat. Reservoir Characterization of Habiganj Gas Field. International Journal of Oil, Gas and Coal Engineering. Vol. 1, No. 1, 2013, pp. 7-15. doi: 10.11648/j.ogce.20130101.12

\begin{abstract}
Although Habiganj Gas Field is one of the best gas field with good reservoir characteristics but it is least analyzed. This paper presents the critical view on four wells (HBJ -7, HBJ-8, HBJ-9, and HBJ-10) of upper gas sand using wire line log data for further development. It is found from the analyses data that a thick zone ( $40 \mathrm{~m}$, depth between 1410 to $1450 \mathrm{~m})$ of Bokabil Formation in well no. $8 \mathrm{HBJ}-8$ shows greater porosity ( $>43 \%)$ and permeability $(5-16 \mathrm{D})$ which may be the indicators of a big fracture zone within the reservoir that yet to be noticed. The similar phenomenon also observed in HBJ-9 with laser thickness. The consistency in porosity and permeability of different wells shows that the reservoir of Habiganj Gas Field is well-sorted. However the stratigraphical model shows that the structure of Habiganj Gas field is almost symmetrical and trending SSE, which supports the structure of Surma Basin. The multi-log 3-D model shows both lithology and stratigraphy, which also resemblance with the lithology and stratigraphy of the Surma Basin.
\end{abstract}

Keywords: Habiganj Gas Field, Surma Basin, Reservoir Characterization, Porosity, Permeability

\section{Introduction}

Reservoir characterization is essential for a sound petroleum management purposing of improving estimation of reserves and making decisions regarding the development of the field. Characterizing a reservoir includes lithology, porosity, permeability, temperature etc. There are many different methods for determining petrophysical characteristics have been published in the literatures for but there is no single method that yields the most reliable answer. The accuracy of the reservoir characterization depends on the availability and reliability of data whereas there are many limitations in data acquisition.

A model of a reservoir that incorporates all the characteristics of the reservoir that is pertinent to its ability to store hydrocarbons. Moreover, a model with a specific volume incorporates all the geologic characteristics of the reservoir and such models are used to quantify characteristics within the subsurface volume. These attributes include the structural shape and thicknesses of the formations within the subsurface volume, their lithology, and the porosity and permeability distributions. The model result can detect heterogeneity of the reservoir. However, the two parameters; porosity and permeability are stable in the near-geologic timeframe and do not change due to the movement of fluids or gases through any of the formations pore spaces.

Habiganj Gas Field is located in the north-eastern part of Bangladesh, lies in Madhabpur Upazila under Habiganj district (Figure 1). From the gross estimation, it is found that the upper gas sand of this field lies at depth of $1320 \mathrm{~m}$ to $1550 \mathrm{~m}$. Reservoir characters of this gas field preliminarily analyzed using empirical method by [1], but there is no detailed analysis yet to be done. We believe that it is one of the least analyzed structures in term of reservoir characterization and has an appeal to assessment its properties. In this paper we tried to i) figure out some distinguishing features which yet to be known using well $\log$ data of the gas field, iii) identify lithology and stratigraphy of different wells based on log data, iv) to analyze changes of different properties with depth of different wells, and v) visualize the entire gas field in three dimensional model.

\section{Geology}

The Surma Basin is a dynamically subsiding [2] sub-basin of the Bengal Basin situated in the northeastern part of Bangladesh. Habiganj Gas Field lies in the southern part of the Surma basin. However, the Aeromagnetic explanation plot [3] indicates a steady deepening of basement towards the center of the basin and also reveals subsurface synclinal features and faults within the basin. Its 
topography is predominantly flat with some north-south trending ridges of twenty to several hundred meters elevation present in the north-eastern border. The thickness of sedimentary strata in the Sylhet Basin ranges from about 13 to $17 \mathrm{~km}$ has been estimated by some workers [4,5]. Much of these strata are Neogene in age [2]. The sedimentary strata in the Sylhet Basin are believed to have been deposited in a deltaic to shallow marine environment [6]. Mostly all of the discovered gas/oil fields have been found within the Bokabil/Bhuban Formation of the Sylhet Basin. However, upper sand zone is relative loose and is disturbing the oil/gas production.

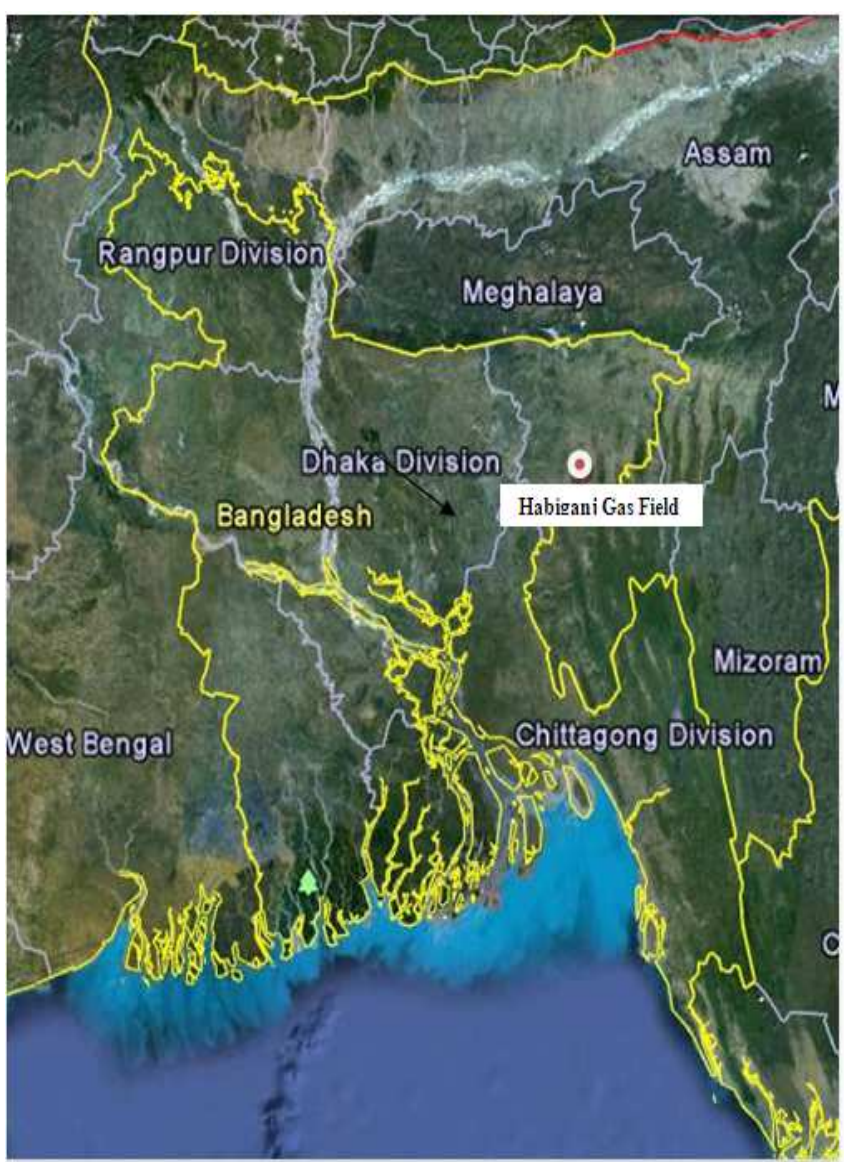

Fig. 1: Location map of Habiganj Gas Field, Bangladesh (downloaded from Google Earth)

\section{Methodology}

Reservoir characterization involves the integration of a vast amount of data from seismic surveys, from geophysical well logs, and from geological samples. In this study, firstly we calculate porosity and permeability of different formation of reservoir from well $\log$ data (Appendix-1-3) collected from Bangladesh Gas Field Company Limited (BGFCL) with kind permission from Bangladesh Oil Gas and Mineral Corporation (Petrobangla).

\subsection{Data Calculation}

Formation porosity was determined from the density log and neutron $\log$

\subsubsection{Porosity Determination}

Porosity from density Log

The bulk density is the overall gross or weighted-average density of a unit of the formation. It can be expressed by

$$
\rho_{\mathrm{b}}=\phi \rho_{\mathrm{f}}+(1-\phi) \rho_{\mathrm{ma}}
$$

Solving for porosity yields,

$$
\phi_{\mathrm{D}}=\left(\rho_{\mathrm{m}}-\rho_{\mathrm{b}}\right) /\left(\rho_{\mathrm{ma}}-\rho_{\mathrm{f}}\right)
$$

Where, $\rho_{\mathrm{ma}}$ is the densityof the rock matrix, $\rho_{\mathrm{b}}$ is the bulk density and $\rho_{\mathrm{f}}$ is the formation fluid density. Common values of $\rho_{\mathrm{ma}}$ are given below in the table 1 .

Table 1: Common values of density of rock matrix and resistivity [7]

\begin{tabular}{cccc}
\hline Rock Type & $\begin{array}{c}\text { Matrix Density } \\
\left(\mathbf{g m} / \mathbf{c m}^{3}\right)\end{array}$ & Rock Type & $\begin{array}{c}\text { Resistivity } \\
(\mathbf{o h m}-\mathbf{m})\end{array}$ \\
\hline $\begin{array}{c}\text { Sand or } \\
\text { Sandstone }\end{array}$ & $1.9-2.65$ & $\begin{array}{c}\text { Compact } \\
\text { formation }\end{array}$ & $300-1000$ \\
Clay & $1.1-1.8$ & $\begin{array}{c}\text { Sandstone and } \\
\text { Limestone } \\
\text { Sand and } \\
\text { Gravel }\end{array}$ & $50-300$ \\
Shale & $2.4-2.8$ & Clay and Shale & $2-100$ \\
Limestone & 2.71 & & \\
Dolomite & 2.87 & & \\
Anhydrite & 2.98 & & \\
\hline
\end{tabular}

Because of the tool's depth, it investigates the invaded zone, and the $\rho_{\mathrm{f}}$ is expressed by

$$
\rho_{\mathrm{f}}=\mathrm{S}_{\mathrm{xo}} \rho_{\mathrm{mf}}+\left(1-\mathrm{S}_{\mathrm{xo}}\right) \rho_{\mathrm{h}}
$$

Where is the mud-filtrate density, $\mathrm{S}_{\mathrm{xo}}$ is the mud-filtrate saturation in invaded zone, and $\rho_{\mathrm{h}}$ is the invaded zone hydrocarbon density. $\rho_{\mathrm{mf}}$ can be approximated according to the mud type. However we use the value of $\rho_{\mathrm{mf}}$ for oil-, water-, and salt-based mud are $0.9,1$, and 1.1 respectively.

\section{Porosity from neutron log}

The neutron log usually measures the hydrogen density of a formation. Neutron porosity $\left(\varphi_{N}\right)$ can computed directly from the logs (Appendix-2). However, investigating the effect of hydrocarbons on the neutron and density logs, [8] have shown that, a range of reasonable and practical values of $\mathrm{S}_{\mathrm{xo}}$, a good approximation is

$$
\phi=\left\{\left(\phi_{\mathrm{D}}^{2}+\phi_{\mathrm{N}}^{2}\right) / 2\right\}^{1 / 2}
$$

\subsubsection{Permeability Determination}

Permeability value were calculated using empirical models are given below- 
Empirical Models

A more general empirical relationship, proposed by [9] is

$$
\mathrm{K}=\mathrm{C} \phi^{\mathrm{x}} /\left(\mathrm{S}_{\mathrm{wi}}\right)^{\mathrm{y}}
$$

The most two common relationships with which permeability can be estimated from porosity and irreducible water saturation derived from equations 3.6 and 3.7 from well logs are according to $[10,11]$ respectively

$$
\begin{aligned}
& \mathrm{K}^{1 / 2}=250\left(\phi^{3} / \mathrm{S}_{\mathrm{wi}}\right) \\
& \mathrm{K}^{1 / 2}=100\left(\phi^{2.25} / \mathrm{S}_{\mathrm{wi}}\right)
\end{aligned}
$$

Where, $\mathrm{K}$ is permeability (md), $\varphi$ is porosity, and $\mathrm{S}_{\mathrm{wi}}$ is irreducible water saturation

\subsection{Lithology Determination}

Lithology of different strata is determined on the basis of reference values of density, gamma ray and resistivity of different rock types. For example, if the density of a rock matrix is 2.19 and the value of resistivity is 100, than the rock matrix will be Sandstone. However, the common values of density and resistivity (Table 1), of different rock matrix were used to established lithology of the field.

\subsection{Data Analysis and Modeling}

We analyses the log data on the basis of porosity and permeability with depth variation as described in previous section (3.1). After calculation, we modeled the Habiganj Gas Field using reservoir modeling software Rock Works $14^{\circledR}$.

\section{Result and Discussion}

Well log data of four wells of Habiganj Gas Field (HBJ-7, HBJ-8, HBJ-9, and HBJ-10) were analyzed to identifying the prospective reservoir formation. We mainly analyzed the Upper gas sand layer of the gas field. The upper gas sand layer lies at a depth from $1320 \mathrm{~m}$ to $1550 \mathrm{~m}$ [12]. According to our data analysis, the Habiganj Gas Field has mostly Sandstone and Shale and they belong to the Bhuban and Bokabil formation of Surma Group of Miocene-Pliocene age [13]. Most of the analyzed well shows a consistent value of porosity (30-35\%) and permeability (2000-3000 $\mathrm{mD})$ with some exceptions. The average porosity of HBJ-7 lies between 30 to $35 \%$ and average permeability lies between $3000 \mathrm{mD}$ to $4000 \mathrm{mD}$. A major decrease of porosity and permeability occur near about the depth of $1375 \mathrm{~m}$ and a major increase of porosity and permeability occur near about the depth of $1400 \mathrm{~m}$. For HBJ-8, average porosity lies between 35 to $45 \%$ and average permeability lies between $8400 \mathrm{mD}$ to $12600 \mathrm{mD}$. A major decrease of porosity and permeability occur near about the depth of $1470 \mathrm{~m}$ and a major increase of porosity $y$ and permeability occur near about the depth of $1415 \mathrm{~m}$. In HBJ-9, average porosity ranges between 30 to $40 \%$ and average permeability between $4000 \mathrm{mD}$ to $8000 \mathrm{mD}$. A major decrease of porosity and permeability occur near about the depth of $1400 \mathrm{~m}$ and a major increase of porosity and permeability occur near about the depth of $1420 \mathrm{~m}$. average porosity lies between 30 to $35 \%$ and average permeability lies between $500 \mathrm{mD}$ to $2000 \mathrm{mD}$ for HBJ-10. A major decrease of porosity and permeability occur near about the depth of $1410 \mathrm{~m}$ and a major increase of porosity and permeability occur near about the depth of $1500 \mathrm{~m}$. These findings are consistence with the previous result (Imam, 2005). However, the Figs. 2 and 3 are evident that there is a thick ( $40 \mathrm{~m}$, depth between 1410 to $1450 \mathrm{~m}$ ) sand layer of HBJ-8 with abnormal value of porosity $(>43 \%)$ and permeability $(>10$ D) which indicative for reservoir fracture [14]. Moreover, HBJ-9 also shows two prospective layers with $30-40 \%$ porosity and greater permeability (8000-16000 $\mathrm{mD})$. However, these two layers have thickness $\sim 15 \mathrm{~m}$ and $\sim 20 \mathrm{~m}$, respective (Fig. 2).

Characterizing a reservoir is very complicated with little data. By this, only approximation of the performance of the reservoir can be done, not the exact prediction. From the stratigraphical model (Fig.3) it is clear that the structure of Habiganj Gas field is almost symmetrical and trending SSE, which supports the structure of Surma Basin. The multi-log 3-D model shows both lithology and stratigraphy, which resemblance with the lithology and stratigraphy of Surma Basin. Again, the correlation between wells (Fig.4) shows that there is continuity among different layers of reservoir of different stratigraphy. For example, if we connect the layers of Bokabil formation of different wells, it shows a continuous layer of Bokabil formation among wells.

\section{Conclusion}

Characterizing a gas reservoir is a complex procedure and in most of the cases the gas reservoir are associated with heterogeneity. Though the data is not sufficient for modeling a big and an excellent reservoir like Habiganj Gas Field, however we try to understand the nature of Habiganj Gas Field. Modeling results indicates that there are some remarkable features like faults in the upper sand land of Habiganj Gas Field that was unrevealed. Moreover, 3-D model can predict and maintain the reservoir performance. 


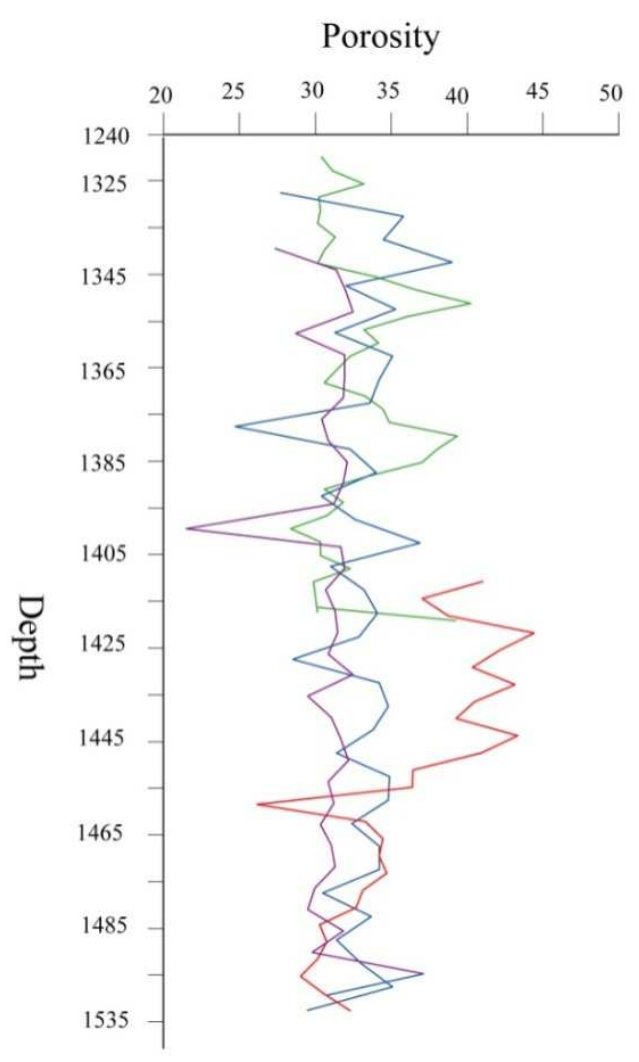

a.

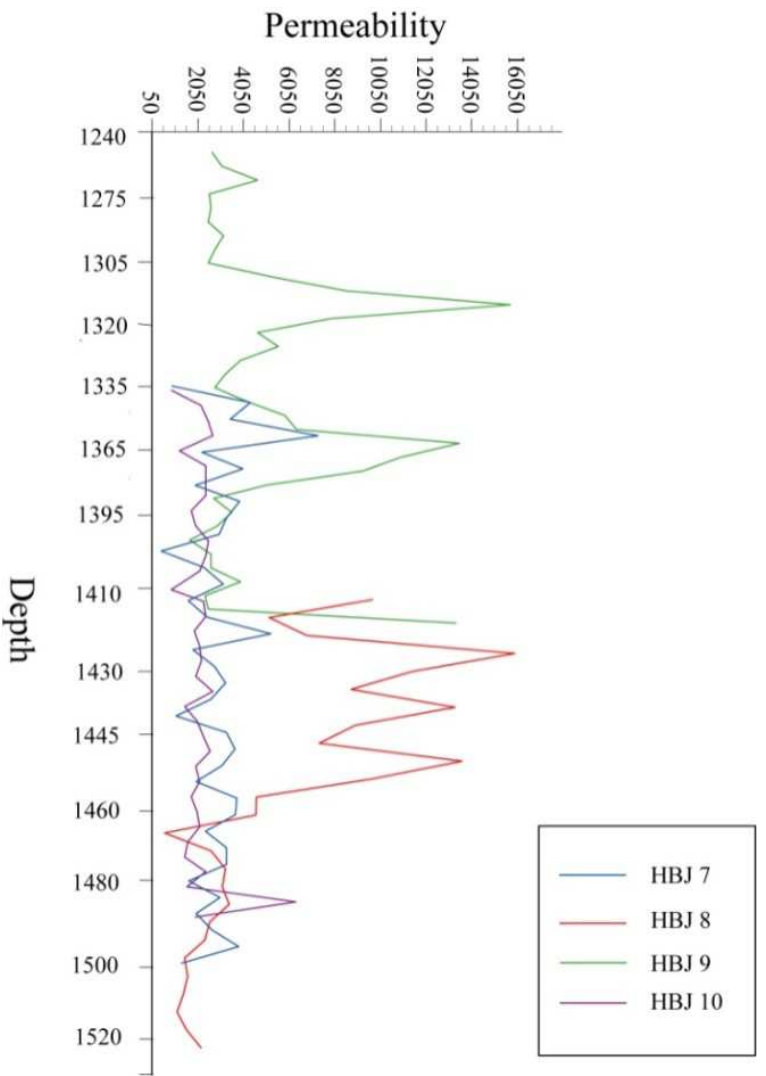

b.

Fig. 2: a) Porosity and b) permeability of different wells of Habiganj Gas Field

Top

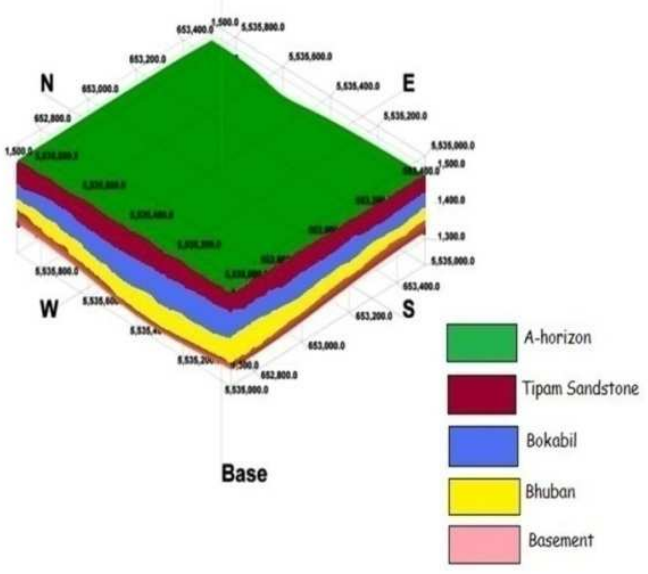

a
Top
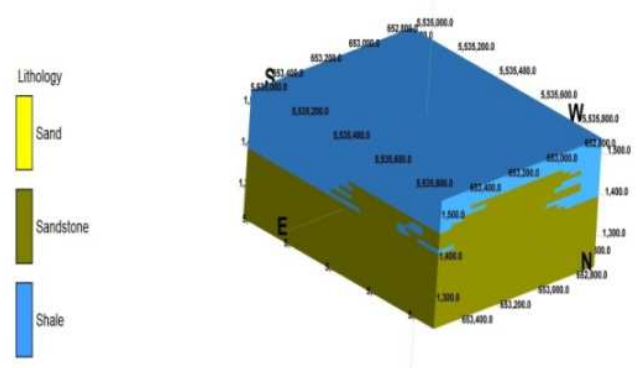

Base

Fig. 3: a) Stratigraphic and b) Lithological model of Habiganj Gas Field 


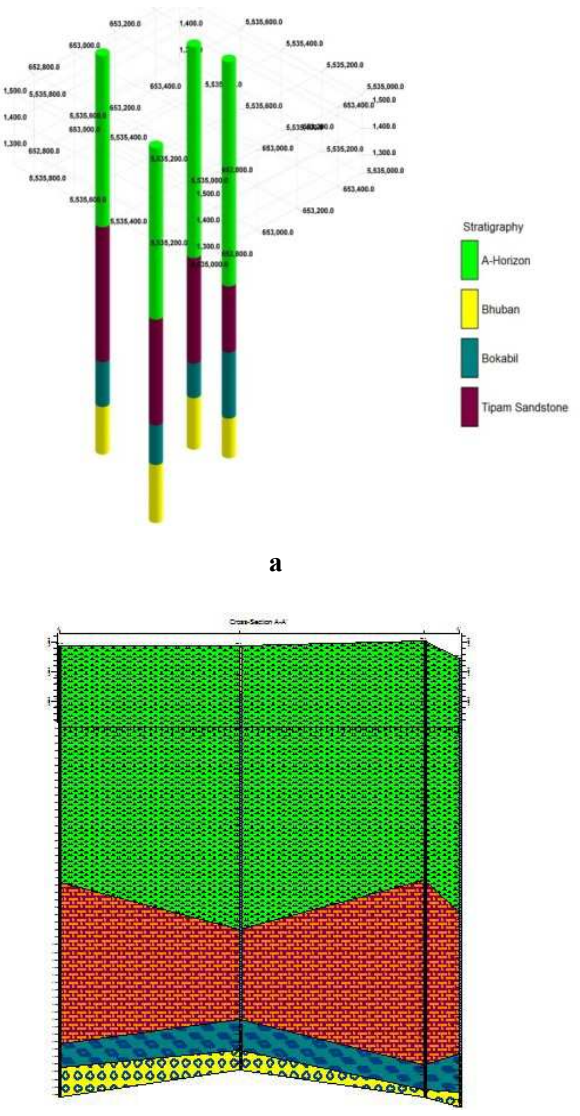

b

Fig. 4: a) Multi-log $3 d$ (stratigraphy) and b) Section straight-line correlations

\section{Appendix 1}

Log data of $\mathrm{HBJ}-7$

\begin{tabular}{cccc}
\hline Depth, $\mathbf{m}$ & Density (gm/cc) & $\begin{array}{c}\text { Resistivity } \\
\text { (ohm-m) }\end{array}$ & Neutron (\%) \\
\hline 1325 & 2.193633 & 100 & 21 \\
1330 & 2.059563 & 150 & 25 \\
1335 & 2.081169 & 200 & 21 \\
1340 & 2.0065 & 55 & 39 \\
1345 & 2.121742 & 30 & 31 \\
1350 & 2.067454 & 200 & 27 \\
1355 & 2.133205 & 65 & 21 \\
1360 & 2.070617 & 250 & 21 \\
1365 & 2.085613 & 100 & 24 \\
1370 & 2.095713 & 90 & 24 \\
1375 & 2.242981 & 50 & 31 \\
1380 & 2.11738 & 300 & 22 \\
1385 & 2.089728 & 300 & 25 \\
1390 & 2.147765 & 100 & 22 \\
\hline
\end{tabular}

\begin{tabular}{|c|c|c|c|}
\hline Depth, m & Density (gm/cc) & $\begin{array}{c}\text { Resistivity } \\
\text { (ohm-m) }\end{array}$ & Neutron (\%) \\
\hline 1395 & 2.112166 & 270 & 19 \\
\hline 1400 & 2.040951 & 205 & 31 \\
\hline 1405 & 2.137702 & 100 & 22 \\
\hline 1410 & 2.102135 & 260 & 21 \\
\hline 1415 & 2.086941 & 300 & 27 \\
\hline 1420 & 2.107127 & 190 & 22 \\
\hline 1425 & 2.179099 & 90 & 30 \\
\hline 1430 & 2.085613 & 250 & 24 \\
\hline 1435 & 2.075928 & 50 & 39 \\
\hline 1440 & 2.091675 & 390 & 21 \\
\hline 1445 & 2.132152 & 100 & 17 \\
\hline 1450 & 2.07368 & 180 & 26 \\
\hline 1455 & 2.075454 & 300 & 24 \\
\hline 1460 & 2.115721 & 160 & 24 \\
\hline 1465 & 2.085613 & 300 & 24 \\
\hline 1470 & 2.085613 & 200 & 24 \\
\hline 1475 & 2.147359 & 330 & 16 \\
\hline 1480 & 2.094241 & 200 & 30 \\
\hline 1485 & 2.132152 & 195 & 17 \\
\hline 1490 & 2.1055 & 40 & 33 \\
\hline 1495 & 2.071558 & 125 & 33 \\
\hline 1500 & 2.16374 & 120 & 21 \\
\hline
\end{tabular}

Log data of HBJ-8

\begin{tabular}{cccc}
\hline Depth, m & density \% & $\begin{array}{c}\text { Resistivity } \\
\text { (ohm-m) }\end{array}$ & Neutron (\%) \\
\hline 1400 & 35 & 2.6 & 51 \\
1405 & 28 & 2.6 & 48 \\
1410 & 30 & 2.6 & 50 \\
1415 & 40 & 2.6 & 54 \\
1420 & 38 & 2.6 & 51 \\
1425 & 36 & 2.7 & 49 \\
1430 & 38 & 2.6 & 53 \\
1435 & 35 & 2.6 & 50 \\
1440 & 33 & 2.6 & 49 \\
1445 & 37 & 2.6 & 54 \\
1450 & 30 & 2.7 & 54 \\
1455 & 26 & 2.8 & 48 \\
1460 & 24 & 3 & 49 \\
1465 & 24 & 5 & 30 \\
1470 & 21 & 3.6 & 45 \\
1475 & 21 & 4 & 47 \\
\hline
\end{tabular}




\begin{tabular}{cccc}
\hline Depth, $\mathbf{m}$ & density \% & $\begin{array}{c}\text { Resistivity } \\
\text { (ohm-m) }\end{array}$ & Neutron (\%) \\
\hline 1480 & 20 & 4.1 & 47 \\
1485 & 22 & 4.4 & 47 \\
1490 & 18 & 5.3 & 46 \\
1495 & 16 & 6.8 & 46 \\
1500 & 38 & 40 & 24 \\
1505 & 40 & 81 & 22 \\
1510 & 40 & 110 & 20 \\
1515 & 39 & 105 & 18 \\
1520 & 34 & 28 & 30 \\
1525 & 35 & 25 & 33 \\
\hline
\end{tabular}

\begin{tabular}{llll}
\hline 1395 & 2.24 & 3.7 & 36 \\
1400 & 2.24 & 3.6 & 36 \\
1405 & 2.22 & 3.7 & 39 \\
1410 & 2.24 & 3.8 & 35 \\
1415 & 2.25 & 3.6 & 36 \\
1420 & 1.96 & 3.3 & 40 \\
\hline
\end{tabular}

\begin{tabular}{|c|c|c|c|}
\hline \multicolumn{4}{|c|}{ Log data of HBJ-9 } \\
\hline Depth, m & $\begin{array}{l}\text { density } \\
\text { (gm/cc) }\end{array}$ & $\begin{array}{c}\text { Resistivity } \\
\text { (ohm-m) }\end{array}$ & Neutron (\%) \\
\hline 1250 & 2.26 & 7.5 & 37 \\
\hline 1255 & 2.25 & 6.7 & 38 \\
\hline 1260 & 2.18 & 6.8 & 39 \\
\hline 1265 & 2.27 & 5.8 & 37 \\
\hline 1270 & 2.24 & 5.1 & 36 \\
\hline 1275 & 2.25 & 4.8 & 36 \\
\hline 1280 & 2.2 & 4.4 & 36 \\
\hline 1285 & 2.25 & 4.4 & 37 \\
\hline 1290 & 2.25 & 4.2 & 36 \\
\hline 1295 & 2.15 & 4 & 39 \\
\hline 1300 & 2.1 & 3.6 & 42 \\
\hline 1305 & 1.97 & 3.4 & 43 \\
\hline 1310 & 2.12 & 3.2 & 42 \\
\hline 1315 & 2.18 & 3.1 & 39 \\
\hline 1320 & 2.16 & 3 & 40 \\
\hline 1325 & 2.22 & 3 & 39 \\
\hline 1330 & 2.24 & 3.1 & 38 \\
\hline 1335 & 2.25 & 3.1 & 37 \\
\hline 1340 & 2.2 & 3.1 & 40 \\
\hline 1345 & 2.17 & 3.2 & 41 \\
\hline 1350 & 2.15 & 3.2 & 41 \\
\hline 1355 & 1.99 & 3.1 & 42 \\
\hline 1360 & 2.04 & 3.1 & 42 \\
\hline 1365 & 2.08 & 3.2 & 42 \\
\hline 1370 & 2.16 & 3.3 & 39 \\
\hline 1375 & 2.23 & 3.5 & 36 \\
\hline 1380 & 2.22 & 3.5 & 38 \\
\hline 1385 & 2.2 & 4 & 35 \\
\hline 1390 & 2.31 & 3.8 & 35 \\
\hline
\end{tabular}

\begin{tabular}{|c|c|c|c|}
\hline Depth, m & $\begin{array}{l}\text { Density } \\
\text { (gm/cc) }\end{array}$ & $\begin{array}{c}\text { Resistivity } \\
\text { (ohm-m) }\end{array}$ & Neutron (\%) \\
\hline 1335 & 2.097 & 30 & 24 \\
\hline 1340 & 2.0295 & 45 & 28 \\
\hline 1345 & 1.8925 & 200 & 15 \\
\hline 1350 & 1.871 & 700 & 14 \\
\hline 1355 & 2.069 & 180 & 25 \\
\hline 1360 & 1.899 & 500 & 17 \\
\hline 1365 & 1.894 & 250 & 17 \\
\hline 1370 & 1.8845 & 500 & 14 \\
\hline 1375 & 1.9555 & 100 & 18 \\
\hline 1380 & 1.9405 & 150 & 15 \\
\hline 1385 & 1.899 & 280 & 18 \\
\hline 1390 & 1.8875 & 550 & 14 \\
\hline 1395 & 1.909 & 770 & 14 \\
\hline 1400 & 2.335 & 165 & 20 \\
\hline 1405 & 1.904 & 310 & 16 \\
\hline 1410 & 1.902 & 800 & 17 \\
\hline 1415 & 1.933 & 640 & 17 \\
\hline 1420 & 1.9275 & 550 & 17 \\
\hline 1425 & 1.896 & 500 & 15 \\
\hline 1430 & 1.9165 & 920 & 15 \\
\hline 1435 & 1.873 & 1500 & 14 \\
\hline 1440 & 1.97 & 550 & 17 \\
\hline 1445 & 1.9175 & 850 & 16 \\
\hline 1450 & 1.911 & 330 & 16 \\
\hline 1455 & 1.8875 & 850 & 16 \\
\hline 1460 & 1.9215 & 300 & 15 \\
\hline 1465 & 1.892 & 300 & 14 \\
\hline 1470 & 1.963 & 30 & 20 \\
\hline 1475 & 2.0135 & 80 & 27 \\
\hline 1480 & 1.9085 & 110 & 17 \\
\hline 1485 & 2.0195 & 120 & 24 \\
\hline 1490 & 2.001 & 140 & 21 \\
\hline 1495 & 1.899 & 300 & 17 \\
\hline 1500 & 2.318 & 30 & 32 \\
\hline 1505 & 1.871 & 33 & 31 \\
\hline 1510 & 2.0515 & 26 & 29 \\
\hline
\end{tabular}


Appendix 2

Data Analysis of HBJ-7

\begin{tabular}{|c|c|c|c|c|c|}
\hline & \multicolumn{2}{|c|}{ Porosity } & \multicolumn{2}{|c|}{ Permeability } & \multirow[b]{2}{*}{ Lithology } \\
\hline $\begin{array}{l}\text { Depth, } \\
\text { m }\end{array}$ & $\varphi_{D} \%$ & $\varphi_{D-N} \%$ & $\begin{array}{c}\text { KTixier, } \\
\text { mD }\end{array}$ & $\begin{array}{c}\text { KTimur, } \\
\mathrm{mD}\end{array}$ & \\
\hline 1325 & 33 & 27.7 & 1093.011 & 1202.26 & sandstone \\
\hline 1330 & 44 & 35.8 & 5126.002 & 3831.458 & sandstone \\
\hline 1335 & 44 & 34.5 & 4098.62 & 3239.725 & sandstone \\
\hline 1340 & 39 & 39 & 8590.683 & 5643.529 & sandstone \\
\hline 1345 & 33 & 32 & 2629.128 & 2322.142 & sandstone \\
\hline 1350 & 42 & 35.3 & 4728.429 & 3606.348 & sandstone \\
\hline 1355 & 39 & 31.3 & 2304.873 & 2103.853 & sandstone \\
\hline 1360 & 45 & 35.1 & 4576.456 & 3519.062 & sandstone \\
\hline 1365 & 42 & 34.2 & 3910.188 & 3127.362 & sandstone \\
\hline 1370 & 41 & 33.6 & 3508.688 & 2883.292 & sandstone \\
\hline 1375 & 16 & 24.7 & 550.0749 & 718.367 & sandstone \\
\hline 1380 & 40 & 32.3 & 2762.124 & 2409.697 & sandstone \\
\hline 1385 & 41 & 34 & 3742.208 & 3026.049 & sandstone \\
\hline 1390 & 37 & 30.4 & 1941.674 & 1849.96 & sandstone \\
\hline 1395 & 42 & 32.6 & 2928.376 & 2517.678 & sandstone \\
\hline 1400 & 42 & 36.9 & 6175.179 & 4405.723 & sandstone \\
\hline 1405 & 38 & 31 & 2187.113 & 2022.709 & sandstone \\
\hline 1410 & 42 & 33.2 & 3271.718 & 2735.973 & sandstone \\
\hline 1415 & 40 & 34.1 & 3855.303 & 3094.382 & sandstone \\
\hline 1420 & 41 & 32.9 & 3096.876 & 2625.565 & sandstone \\
\hline 1425 & 27 & 28.5 & 1319.208 & 1384.411 & sandstone \\
\hline 1430 & 42 & 34.2 & 3910.188 & 3127.362 & sandstone \\
\hline 1435 & 30 & 34.8 & 4330.464 & 3376.221 & sandstone \\
\hline 1440 & 43 & 33.8 & 3664.853 & 2979.013 & sandstone \\
\hline 1445 & 41 & 31.4 & 2333.183 & 2123.203 & sandstone \\
\hline 1450 & 42 & 34.9 & 4433.223 & 3436.131 & sandstone \\
\hline 1455 & 43 & 34.8 & 4351.964 & 3388.785 & sandstone \\
\hline 1460 & 39 & 32.4 & 2814.138 & 2443.65 & sandstone \\
\hline 1465 & 42 & 34.2 & 3910.188 & 3127.362 & sandstone \\
\hline 1470 & 42 & 34.2 & 3910.188 & 3127.362 & sandstone \\
\hline 1475 & 40 & 30.5 & 1951.12 & 1856.705 & sandstone \\
\hline 1480 & 37 & 33.7 & 3564.951 & 2917.898 & sandstone \\
\hline 1485 & 41 & 31.4 & 2333.183 & 2123.203 & sandstone \\
\hline 1490 & 33 & 33 & 3152.998 & 2661.171 & sandstone \\
\hline 1495 & 37 & 35.1 & 4532.061 & 3493.428 & sandstone \\
\hline 1500 & 36 & 29.5 & 1599.372 & 1599.529 & sandstone \\
\hline
\end{tabular}

Data Analysis of HBJ-8

\begin{tabular}{|c|c|c|c|c|c|}
\hline \multicolumn{3}{|c|}{ Porosity } & \multicolumn{2}{|c|}{ Permeability } & \multirow[b]{2}{*}{ Lithology } \\
\hline Depth, m & $\varphi_{D} \%$ & \multicolumn{2}{|r|}{$\begin{array}{c}\text { KTixier, } \\
\text { mD }\end{array}$} & $\begin{array}{l}\text { KTimur, } \\
\text { mD }\end{array}$ & \\
\hline 1400 & 43.73786 & \multicolumn{2}{|r|}{9921.706} & 5488.076 & Shale \\
\hline 1405 & 39.29377 & \multicolumn{2}{|r|}{5261.549} & 3388.581 & Shale \\
\hline 1410 & 41.23106 & \multicolumn{2}{|r|}{6962.868} & 4207.959 & Shale \\
\hline 1415 & 47.51842 & \multicolumn{2}{|r|}{16315.98} & 7969.669 & Shale \\
\hline 1420 & 44.97221 & \multicolumn{2}{|r|}{11724.84} & 6220.29 & Shale \\
\hline 1425 & 42.99419 & \multicolumn{2}{|r|}{8951.582} & 5080.485 & Shale \\
\hline 1430 & 46.11399 & \multicolumn{2}{|r|}{13628.17} & 6963.195 & Shale \\
\hline 1435 & 43.15669 & \multicolumn{2}{|r|}{9156.516} & 5167.471 & Shale \\
\hline 1440 & 41.7732 & \multicolumn{2}{|r|}{7530.568} & 4462.735 & Shale \\
\hline 1445 & 46.28715 & \multicolumn{2}{|r|}{13938.11} & 7081.631 & Shale \\
\hline 1450 & 43.68066 & & 9844.112 & 5455.854 & Shale \\
\hline 1455 & 38.60052 & & 4688.136 & 3127.739 & Shale \\
\hline 1460 & 38.58108 & & 4673.992 & 3120.659 & Shale \\
\hline 1465 & 27.16616 & & 569.6532 & 643.707 & Shale \\
\hline 1470 & 35.1141 & & 2656.627 & 2042.812 & Shale \\
\hline 1475 & 36.40055 & & 3296.773 & 2401.857 & Shale \\
\hline 1480 & 36.11786 & & 3146.108 & 2319.053 & Shale \\
\hline 1485 & 36.69469 & & 3459.876 & 2490.438 & Shale \\
\hline 1490 & 34.9285 & & 2573.481 & 1994.67 & Shale \\
\hline 1495 & 34.43835 & & 2364.261 & 1871.768 & Shale \\
\hline 1500 & 31.7805 & & 1460.177 & 1304.021 & sandstone \\
\hline 1505 & 32.28002 & & 1603.41 & 1398.826 & sandstone \\
\hline 1510 & 31.62278 & & 1417.234 & 1275.15 & sandstone \\
\hline 1515 & 30.37269 & & 1112.604 & 1063.496 & sandstone \\
\hline 1520 & 32.06244 & & 1539.646 & 1356.894 & sandstone \\
\hline 1525 & 34.0147 & & 2195.035 & 1770.36 & sandstone \\
\hline & & Data A & Analysis of $H$ & BJ-9 & \\
\hline & Porosi & & Perm & eability & \\
\hline $\begin{array}{c}\text { Depth, } \\
\text { m }\end{array}$ & $\phi_{D} \%$ & $\varphi_{D-N} \%$ & $\begin{array}{l}\text { KTixier, } \\
\text { mD }\end{array}$ & $\begin{array}{c}\text { KTimur, } \\
\mathrm{mD}\end{array}$ & Lithology \\
\hline 1250 & 26.3 & 32.1 & 2673.703 & 2351.608 & Shale \\
\hline 1255 & 26.9 & 32.9 & 3108.225 & 2632.778 & Shale \\
\hline 1260 & 31 & 35.2 & 4664.07 & 3569.471 & Shale \\
\hline 1265 & 25.7 & 31.9 & 2557.018 & 2274.209 & Shale \\
\hline 1270 & 27.5 & 32 & 2634.695 & 2325.829 & Shale \\
\hline 1275 & 26.9 & 31.8 & 2514.044 & 2245.482 & Shale \\
\hline 1280 & 29.8 & 33.1 & 3185.702 & 2681.846 & Shale \\
\hline 1285 & 26.9 & 32.3 & 2796.627 & 2432.237 & Shale \\
\hline 1290 & 26.9 & 31.8 & 2514.044 & 2245.482 & Shale \\
\hline
\end{tabular}




\begin{tabular}{|c|c|c|c|c|c|}
\hline \multirow[b]{2}{*}{1295} & \multicolumn{2}{|c|}{ Porosity } & \multicolumn{2}{|c|}{ Permeability } & \multirow[b]{2}{*}{ Shale } \\
\hline & 32.7 & 36 & 5323.437 & 3941.614 & \\
\hline 1300 & 35.7 & 39 & 8544.41 & 5620.715 & Shale \\
\hline 1305 & 43.3 & 43.1 & 15731.81 & 8884.112 & Shale \\
\hline 1310 & 34.5 & 38.4 & 7870.11 & 5284.642 & Shale \\
\hline 1315 & 31 & 35.2 & 4664.07 & 3569.471 & Shale \\
\hline 1320 & 32.2 & 36.3 & 5580.175 & 4083.343 & Shale \\
\hline 1325 & 28.7 & 34.2 & 3920.762 & 3133.703 & Shale \\
\hline 1330 & 27.5 & 33.2 & 3247.058 & 2720.492 & Shale \\
\hline 1335 & 27 & 32.3 & 2796.627 & 2432.237 & Shale \\
\hline 1340 & 29.8 & 35.3 & 4208.567 & 3594.981 & Shale \\
\hline 1345 & 31.6 & 36.6 & 5862.646 & 4237.407 & Shale \\
\hline 1350 & 32.7 & 37.1 & 6370.735 & 4509.955 & Shale \\
\hline 1355 & 42.1 & 42.1 & 13502.1 & 7921.928 & Sandstone \\
\hline 1360 & 39.2 & 40.6 & 10958.86 & 6774.138 & Sandstone \\
\hline 1365 & 36.8 & 39.5 & 9280.548 & 5980.124 & Sandstone \\
\hline 1370 & 32.2 & 35.7 & 5093.085 & 3812.99 & Sandstone \\
\hline 1375 & 28.1 & 32.3 & 2761.865 & 2409.527 & Sandstone \\
\hline 1380 & 28.7 & 33.7 & 3546.634 & 2906.647 & Sandstone \\
\hline 1385 & 29.8 & 32.5 & 2885.2 & 2489.785 & Sandstone \\
\hline 1390 & 23.4 & 29.8 & 1698.522 & 1673.337 & Sandstone \\
\hline 1395 & 27.5 & 32 & 2634.695 & 2325.829 & Sandstone \\
\hline 1400 & 27.5 & 32 & 2634.695 & 2325.829 & Sandstone \\
\hline 1405 & 28.7 & 34.2 & 3920.762 & 3133.703 & Sandstone \\
\hline 1410 & 27.5 & 31.5 & 2370.496 & 2148.619 & Sandstone \\
\hline 1415 & 26.9 & 31.8 & 2514.044 & 2245.482 & Sandstone \\
\hline 1420 & 43.9 & 42 & 13351.66 & 7855.638 & Sandstone \\
\hline
\end{tabular}

Data Analysis of HBJ-10

\begin{tabular}{lccccc}
\hline \multicolumn{4}{c}{ Porosity } & \multicolumn{3}{c}{ Permeability } \\
\hline $\begin{array}{c}\text { Dept } \\
\mathrm{h}, \mathrm{m}\end{array}$ & $\phi_{D} \%$ & $\varphi_{D-N} \%[0,1]$ & KTixier, $\mathrm{mD}$ & $\begin{array}{c}\text { KTimur, } \\
\mathrm{mD}\end{array}$ & Lithology \\
1335 & 33.5 & 28.3 & 1107.266143 & 1177.754 & sandstone \\
1340 & 37.6 & 32.8 & 2697.89052 & 2296.856 & sandstone \\
1345 & 45.9 & 33.5 & 3079.213259 & 2536.269 & sandstone \\
1350 & 47.2 & 34 & 3340.84 & 2696.232 & sandstone \\
1355 & 35.2 & 29.8 & 1527.158947 & 1498.921 & sandstone \\
1360 & 45.5 & 33.4 & 2977.708777 & 2473.302 & sandstone \\
1365 & 45.8 & 33.4 & 2977.708777 & 2473.302 & sandstone \\
1370 & 46.4 & 33.3 & 2961.680613 & 2463.311 & sandstone \\
1375 & 42.1 & 31.7 & 2178.8899 & 1956.78 & sandstone \\
1380 & 43 & 32.2 & 2411.671159 & 2111.563 & sandstone \\
\hline
\end{tabular}

\begin{tabular}{cccccl}
\hline \multicolumn{5}{c}{ Porosity } & \multicolumn{2}{c}{ Permeability } & \\
\hline 1385 & 45.5 & 33.6 & 3120.450526 & 2561.701 & sandstone \\
1390 & 46.2 & 33.3 & 2961.680613 & 2463.311 & sandstone \\
1395 & 44.9 & 32.6 & 2619.715641 & 2246.757 & sandstone \\
1400 & 19.1 & 20 & 138.4083045 & 247.5923 & sandstone \\
1405 & 45.2 & 33.1 & 2847.172503 & 2391.53 & sandstone \\
1410 & 45.3 & 33.4 & 2977.708777 & 2473.302 & sandstone \\
1415 & 43.5 & 32 & 2339.155765 & 2063.763 & sandstone \\
1420 & 43.8 & 32.7 & 2641.895608 & 2261.009 & sandstone \\
1425 & 45.7 & 32.9 & 2728.076232 & 2316.103 & sandstone \\
1430 & 44.5 & 32.2 & 2411.671159 & 2111.563 & sandstone \\
1435 & 47.1 & 34 & 3340.84 & 2696.232 & sandstone \\
1440 & 41.2 & 30.7 & 1822.166969 & 1711.223 & sandstone \\
1445 & 44.4 & 32.4 & 2521.44081 & 2183.243 & sandstone \\
1450 & 44.8 & 33.1 & 2847.172503 & 2391.53 & sandstone \\
1455 & 46.2 & 33.7 & 3208.24906 & 2615.571 & sandstone \\
1460 & 44.2 & 32.2 & 2411.671159 & 2111.563 & sandstone \\
1465 & 45.9 & 32.6 & 2619.715641 & 2246.757 & sandstone \\
1470 & 41.6 & 31.6 & 2162.631152 & 1945.819 & sandstone \\
1475 & 38.6 & 32.4 & 2496.368425 & 2166.941 & sandstone \\
1480 & 44.9 & 32.7 & 2641.895608 & 2261.009 & sandstone \\
1485 & 38.2 & 31.2 & 1989.073429 & 1827.484 & sandstone \\
1490 & 39.3 & 30.7 & 1810.614558 & 1703.08 & sandstone \\
1495 & 45.5 & 33.3 & 2977.708777 & 2473.302 & sandstone \\
1500 & 31 & 31 & 1925.338008 & 1783.388 & sandstone \\
1505 & 47.2 & 39.2 & 7875.4405 & 5129.46 & sandstone \\
1510 & 36.3 & 32.1 & 2383.874309 & 2093.283 & sandstone \\
\hline & & & & & \\
\hline & & & & & \\
\hline
\end{tabular}

\section{References}

[1] Sultan, J B M Reservoir characterization of Habigonj Gas Field using empirical methods, unpublished B.Sc thesis, 2011, Shahjalal University of Science and Technology, Sylhet, Bangladesh.

[2] Johnson, S Y and Alam, A M N Sedimentation and tectonics of the Sylhet trough, Bangladesh. Geological Society of America Bulletin, 1991.103,1513-1527.

[3] Hunting, Aeromagnetic survey of Bangladesh. Geology and Geophysics Ltd.1980

[4] Evans, P The tectonic framework of Assam, Journal Geological Society of India, 1964. 5, 80-96.

[5] Hiller, $\mathrm{K}$ and Elahi, $\mathrm{M}$ Structural development and hydrocarbon entrapment in the Surma Basin, Bangladesh (northwest Indo-Burman fold belt), Singapore Fifth Offshore Southwest Conference, 1984. 656-663.

[6] Rahman, M. J J , and McCann, T Diagenetic history of the Surma Group sandstones (Miocene) in the Surma Basin, Bangladesh, Journal of Asian Earth Sciences, 2012. 45(2), $65-78$ 
[7] Schlumberger, Oil field Review, 2012, 84-86.

[8] Gaymard, R and Poupon, A Response of neutron and formation density logs in hydrocarbon bearing formations, The Log Analyst, 1968, 9, 3 - 12.

[9] Wyllie, M R J and Rose, W D Some Theoretical Considerations Related to the Quantitative Evaluation of the Physical Characteristics of Reservoir Rock from Electric Log Data, Trans., AIME, 1950, 189, pp105.

[10] Tixier, MP Evaluation of permeability from Electric-Log Resistivity Gradients, Oil \& Gas Journal, 1949. 113.

[11] Timur, AAn investigation of permeability, porosity, and
Residual Water Saturation relationship for sandstone Reservoirs, The Log Analyst, 1968. 9(4),90-123.

[12] Imam, B Energy Resources of Bangladesh, University Grants Commission Publication, 2005, pp280.

[13] Reimann, K.-U., 1993. Geology of Bangladesh. Gebrüder Borntraeger, Berlin. Schlumberger Log Interpretation Principles/Application; Seventh printing, March 1998 (C) Schlumberger 1991; Selley, RElements of Petroleum Geology, 2nd edition, 1998. 470

[14] Laongsakul, P and Dürrast, H Characterization of reservoir fractures using conventional geophysical logging, 2011. 33 (2), 237-246. 